\title{
Duplication of the Gallbladder: A Rare Congenital Anomaly
}

\author{
Madhusudhan KS , Saumya Srivastava $^{2}$, Deep N Srivastava ${ }^{l}$ \\ Department of Radio-diagnosis, All India Institute of Medical Sciences, New Delhi ${ }^{1}$ \\ Department of Microbiology, All India Institute of Medical Sciences, Jodhpur².
}

\begin{abstract}
Biliary tract shows a large number of anatomic variations and duplication of the gallbladder (GB) is a rare congenital anomaly. Although it is very often detected incidentally, it may present with complications due to gall stone disease. Careful identification of this anomaly on imaging is important to prevent any serious surgical complications. We here report a case of duplicated GB in a 26-year-old male, who presented with right hypochondriac pain and detected to have two GB on magnetic resonance imaging $(\mathrm{MRI})$.
\end{abstract}

Keywords: Gallbladder duplication, cholelithiasis, biliary anomalies.

\section{Introduction}

Duplication of the gallbladder (GB) is a rare congenital anomaly of the biliary tract with an incidence of approximately one in 4000 births (1). It may be detected incidentally on imaging done for other reasons or patients may present with symptoms due to any pathology affecting the GB (2). As many surgeries of GB are done laparoscopically, pre-operative identification of biliary anomalies, including duplicated GB is important to prevent inadvertent biliary and arterial injuries and associated morbidity and mortality (3). We report a case of duplicated GB with gall stones in both of them, diagnosed on magnetic resonance imaging (MRI).

\section{Case Report}

A 26-year-old male presented with history of pain in right hypochondrium associated with fever and nausea for four months. There was no history of jaundice, loss of weight or loss of appetite. Clinical examination was unremarkable. Liver function tests were unremarkable, except for mild elevation of serum alkaline phosphatase (253 IU/L; reference: $35-114 \mathrm{IU} / \mathrm{L})$ with normal white blood cell counts and serological studies for viral hepatitis and dengue were negative. Ultrasonography (USG) showed GB full of calculi with posterior shadowing. There was no dilatation of intrahepatic biliary ducts and bile duct was normal. Since the serum alkaline phosphatase was elevated, MRI with magnetic resonance cholangiopancreatography (MRCP) was done to look for bile duct calculi.

MRI showed double gallbladders, which were united at the neck region (Fig. 1). One showed multiple calculi (signal voids on $\mathrm{T} 1$ and T2 weighted images) and the other showed a single calculus with sludge (seen as hyperintense bile on T1-weighted images). The wall was normal. There was no evidence of cholecystitis. Cystic duct could not be visualized. The biliary ducts were normal. A final diagnosis of duplicated GB with cholelithiasis in both sacs

Correspondence: Dr. Madhusudhan KS, Associate Professor, Department of Radio-diagnosis, All India Institute of Medical Sciences, Ansari Nagar, New Delhi - 110029, India. Email: drmadhuks@gmail.com. 


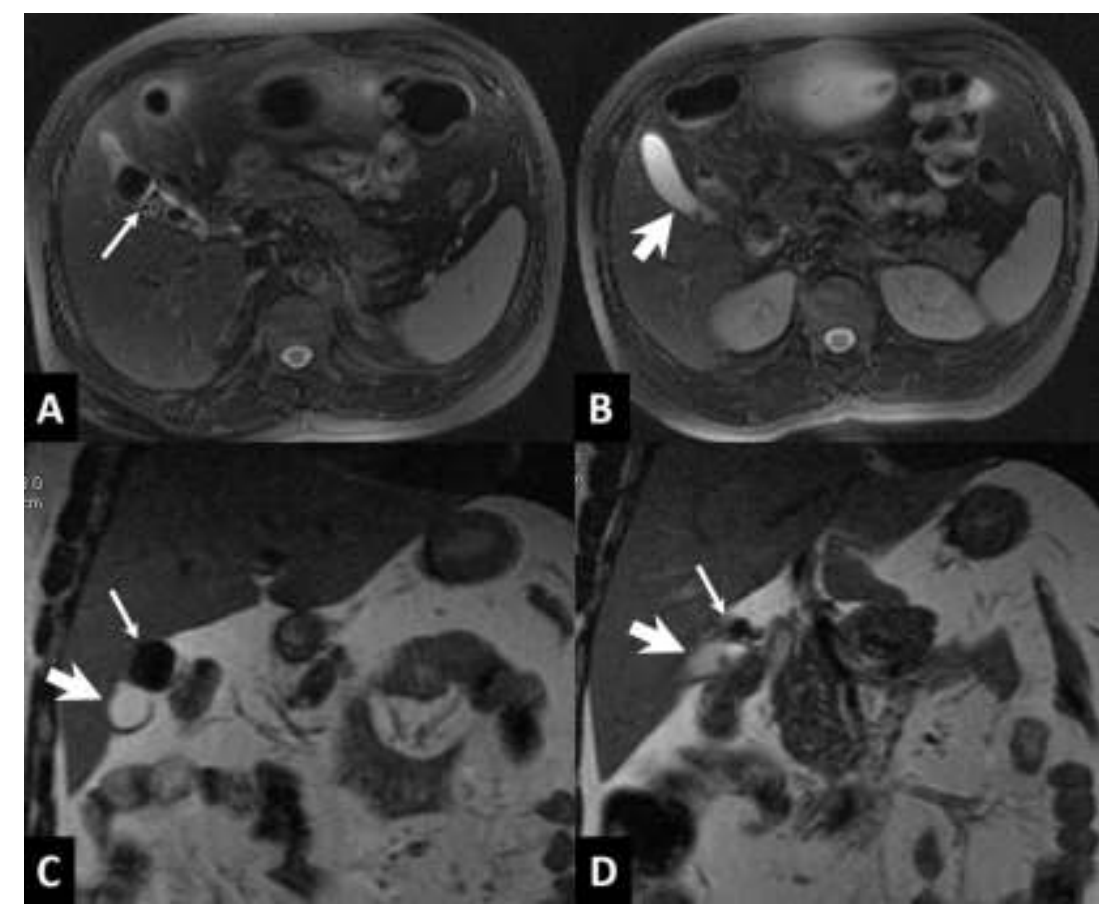

Fig. 1: A \& B: Axial T2-weighted MR images showing two gallbladders, one with multiple calculi (thin arrow in $A$ ) and the other with solitary calculus (thick arrow in B).

C \& D: Coronal T2-weighted MR images showing both gallbladders (thick and thin arrows) with two separate necks and cystic ducts (arrows in D).

was made and the patient was referred for surgery.

\section{Discussion}

Duplication of the GB is an unusual congenital anomaly which occurs due to splitting of cystic primordium of the GB or due to the growth of an accessory bud from the biliary primordium during embryogenesis $(1,4)$. This results in the formation of two epithelium lined sacs which are either partially joined or completely separate from each other.

There are many classifications of duplication of GB. Boyden (1) classified them into two groups based on the presence of two separate cystic ducts. In the first - vesical fellea divisa - the GB is bilobed with common neck and no separate cystic duct. A longitudinal septum or invaginating cleft separates the lumen into two chambers (Fig. 2). In these cases both gall bladders share a common embryological origin (primordium). In the second type-vesical fellea duplex - there are two separate GB and cystic ducts. It has two subtypes - one in which two cystic ducts join together before joining the common bile duct (Y-type) and the other in which two cystic ducts separately join the common bile duct (H-type) (Fig. 3). In the second type a double embryological origin (dual primordium) is considered.

Clinical presentation is non-specific and the incidence of cholelithiasis appears similar to that of general population (5). They may be incidentally detected on imaging. USG shows two cystic structures in the GB fossa (6). However, classification is difficult as cystic duct is usually not identified. Further, the second sac may be missed if there is extensive shadowing due the calculi in one sac. MRI with MRCP will better demonstrate the duplicated GB and its type (7). 


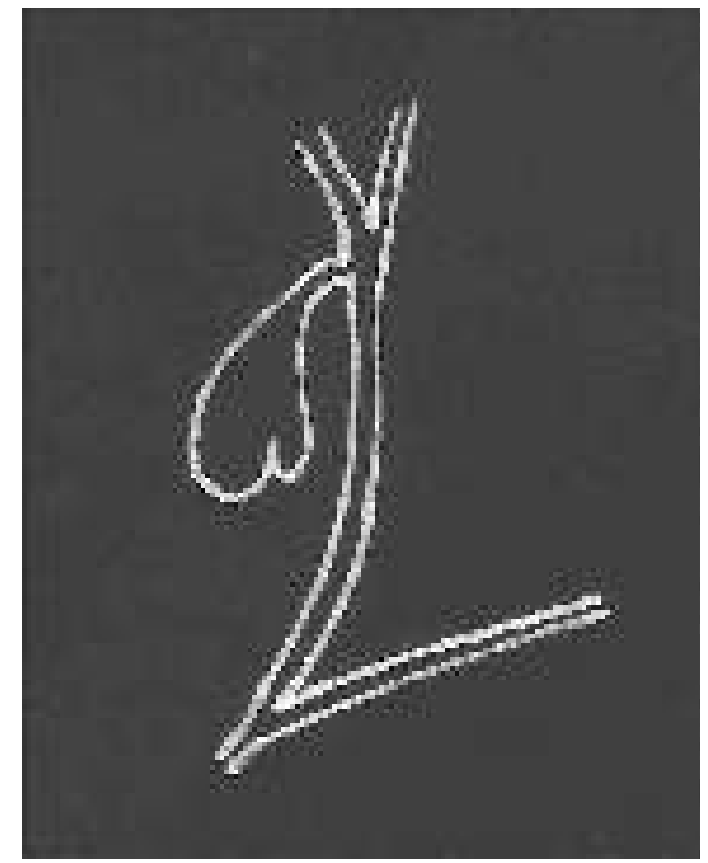

Fig. 2: Diagramatic representation of vesical fellea divisa. The gall bladder (GB) is bilobed with common neck and single cystic duct.

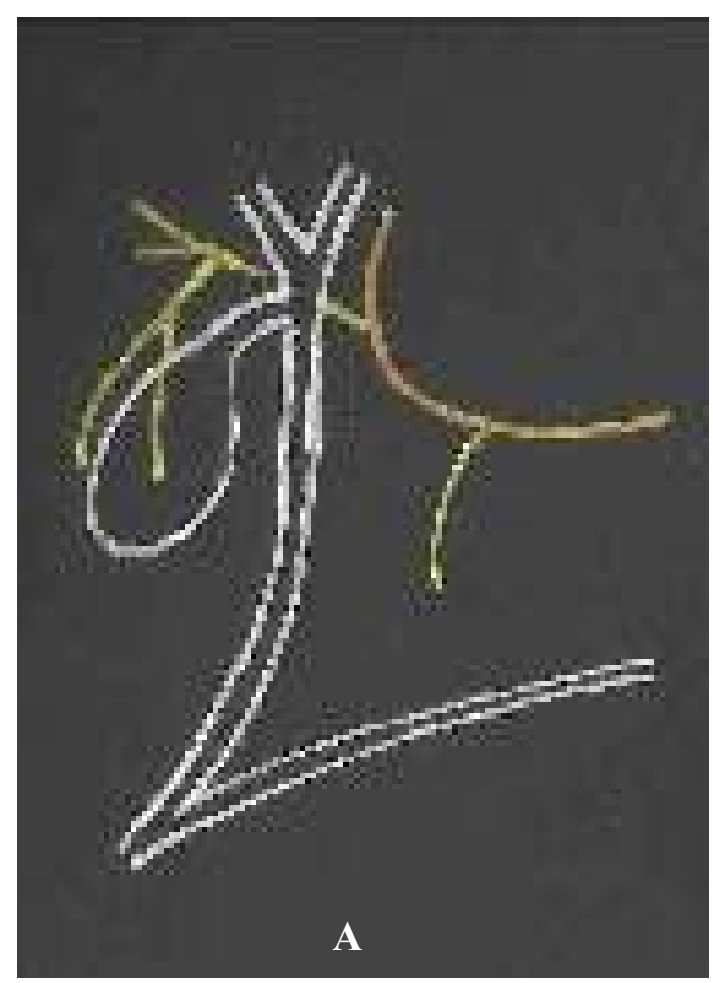

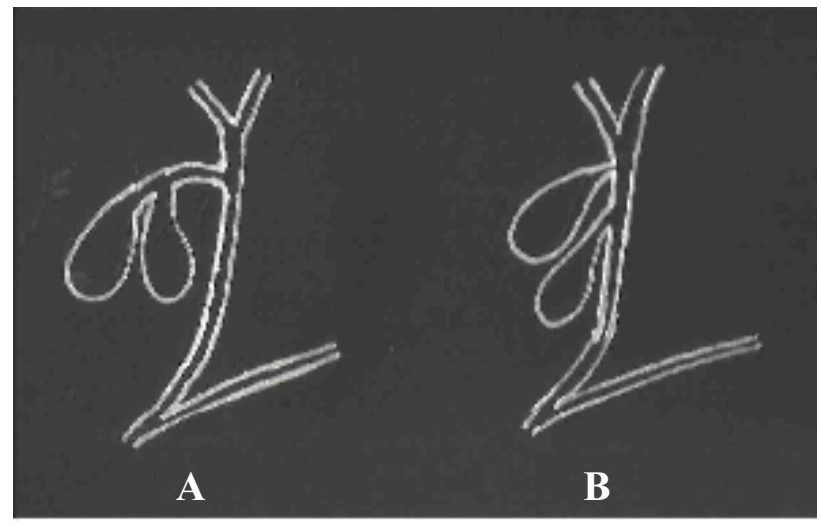

Fig. 3: Diagramatic representation of vesical fellea duplex. There are two separate GB and cystic ducts. In Y type cystic ducts join together before joining common bile duct (CBD) (A) and in H-type the two cystic ducts join CBD separately (B).

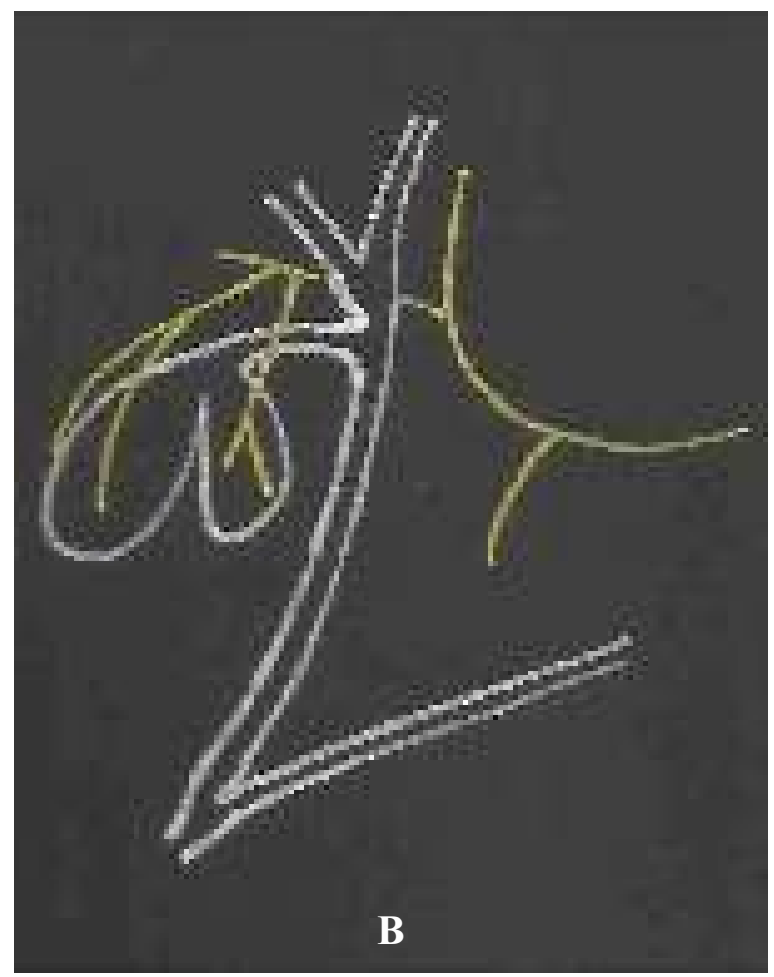

Fig. 4: Diagramatic representation of normal GB with single cystic artery (A). In duplication of GB there may be anatomic variations of hepatic arteries like multiple cystic arteries (B). 
Duplicated GB does not need any treatment unless complicated by calculi or mass. In symptomatic cases, surgery is the treatment of choice. It may be done by open or laparoscopic approaches (8). Pre-operative diagnosis is important to prevent any inadvertent complications because of associated anatomical variations of cystic duct and hepatic arteries (9, 10, 11) (Fig. 4).

In conclusion, duplicated GB is a rare congenital anomaly and mostly asymptomatic. Imaging with USG or MRI is very useful in its detection and classification. Pre-operative diagnosis is helpful in avoiding complications during surgery.

\section{References}

1. Boyden EA (1926). The accessory gallbladder- an embryological and comparative study of aberrant biliary vesicles occurring in man and the domestic mammals. Am J Anat 38: 177-231.

2. Causey MW, Miller S, Fernelius CA, Burgess JR, Brown TA, Newton C (2010). Gallbladder duplication: evaluation, treatment and classification. $J$ Pediatric Surg 45: 443-446.

3. Vijayaraghavan R, Belagavi CS (2006). Double gallbladder with different disease entities: a case report. J Minimal Acc Surg 2: 23-26.

4. Harlaftis N, Gray SW, Olafson RP, et al (1976). Three cases of unsuspected double gallbladder. Am Surg 42: 178-180.
5. Bulus H, Koyuncu A, Coskun A (2012). Preoperative diagnosis of double gallbladder: a case report. Turk J Gastroenterol 23: 172-174.

6. Goiney RC, Schoenecker SA, Cyr DR, Shuman WP, Peters MJ, Cooperberg PL (1985). Sonography of gallbladder duplication and differential considerations. Am J Roentgenol 145: 241-243.

7. Mazziotti S, Minutoli F, Blandino A, Vinci S, Salamone I, Gaeta M (2001). Gallbladder duplication: MR cholangiography demonstration. Abdom Imaging 26: 287-289.

8. Goel A, Srivastava KN, Rana AK (2003). Double gall-bladder - a laparoscopic management. Surg Laparosc Endosc Percutan Tech 13: 348-349.

9. Udelsman R, Sugarbakee PH (1985). Congenital duplication of the gall bladder associated with an anomalous right hepatic artery. Am J Surg 149(6):812-815.

10. Pillay Y (2015). Gall bladder duplication. Intl J Surg Case Rep 11: 18-20.

11. Ramachandran A, Srivastava DN, Gupta AK, Madhusudhan KS (2018). The double trouble: a case of duplicated extrahepatic bile duct with choledochal cyst. Indian $J$ Pediatr (http://doi.org/10.1007/s12098018-2790-1). 